\title{
Non-thermal X-ray emission from young supernova remnants
}

\author{
E. van der Swaluw ${ }^{1,2}$ and A. Achterberg ${ }^{1}$ \\ 1 Astronomical Institute, Utrecht University, PO Box 80000, 3508 TA Utrecht, The Netherlands \\ 2 FOM-Institute for Plasma Physics Rijnhuizen, PO Box 1207, 3430 BE Nieuwegein, The Netherlands
}

Received 21 October 2003 / Accepted 8 March 2004

\begin{abstract}
The Galactic (nucleonic) cosmic-ray spectrum up to the knee $\left(E \sim 10^{15} \mathrm{eV}\right)$ is attributed to acceleration processes that take place near the external shocks around supernova remnants (SNRs). Theoretical predictions based on the theory of diffusive shock acceleration give a similar estimate for the maximum particle energy that can be reached at these shocks: $E \sim 10^{14}-10^{15} \mathrm{eV}$. Electrons with energies $E \sim 10^{14} \mathrm{eV}$ radiate $\mathrm{X}$-ray photons in the $\sim 10-100 \mu \mathrm{G}$ magnetic fields present in many young SNRs. These electrons near the knee give rise to a non-thermal X-ray component in the spectrum of young supernova remnants. Recent observations of SN1006 and G347.3-0.5 confirm this prediction.

We have combined hydrodynamical calculations of the evolution of a young remnant with an algorithm that simultaneously calculates the acceleration of electrons, their radiation losses and the synchrotron spectrum of a young supernova remnant. The electrons are treated using a test-particle approximation.

We give a semi-analytical estimate of the maximum electron energy and typical synchrotron frequencies for young remnants at the end of the free-expansion stage of their evolution. We present spectra of the energy distribution of the electrons in a young supernova remnant, and construct a synchrotron map in the X-ray domain, assuming Bohm diffusion within the remnant and a shock-compressed magnetic field.
\end{abstract}

Key words. ISM: cosmic rays - ISM: supernova remnants - shock waves - acceleration of particles

\section{Introduction}

In this paper we consider the acceleration of electrons at the blast wave surrounding a young supernova remnant. The theory of diffusive shock acceleration (DSA) predicts that nuclei and electrons are accelerated efficiently at this blastwave (see Drury 1983; Blandford \& Eichler 1987; Jones \& Ellison 1991; and Kirk 1994 for a review of DSA).

The nucleonic component of Galactic cosmic rays are thought to be produced in SNRs by this acceleration process. Nuclei can be accelerated up to the energy of the knee $\left(E \sim 10^{15} \mathrm{eV}\right)$ of the Galactic cosmic ray spectrum. At the knee, the slope $s$ of the spectrum steepens from its low-energy value, $s \equiv-\mathrm{d} \ln N(E) / \mathrm{d} \ln E \approx 2.7$, to $s \approx 3.0$, possibly signalling a change in the production process responsible for particles above $10^{15} \mathrm{eV}$, or reacceleration distributed throughout the Galaxy.

Recent discoveries of non-thermal X-ray emission from SNRs seem to suggest that relativistic electrons are also accelerated by SNR shocks to similar energies, $E \approx 10-100 \mathrm{TeV}$. The X-ray spectrum of three SNRs is dominated by nonthermal emission: SN1006 (Koyama et al. 1995), G347.3-0.5 (Koyama et al. 1997; Slane et al. 1999) and G266.2-1.2 (Slane et al. 2001). Furthermore Cas A, Kepler, Tycho and RCW 86 (Allen et al. 1999) also show a non-thermal X-ray component,

Send offprint requests to: E. van der Swaluw, e-mail: swaluw@rijnh.nl but in these three systems there is a significant thermal component below $10 \mathrm{keV}$. In the case of Cas A (Gotthelf et al. 2001), Tycho's remnant (Hwang et al. 2002) and in the remnant of SN1006 (Koyama et al. 1995) there seems to be an outer diffuse rim of synchrotron emission associated with the blast wave.

These observations suggest that young SNRs indeed produce relativistic electrons with a simple power law distribution with slope $s \sim 2.2$ up to an energy of $\sim 10 \mathrm{TeV}$, close to the energy $(\sim 1000 \mathrm{TeV})$ associated with the knee in the nucleonic component of the Galactic cosmic rays. This seems to confirm one of the basic predictions of the theory of DSA. A problem with this simple scenario, which we will not address here, is the fact that no detectable gamma-ray emission is observed from most young remnants, although gamma-rays have been detected from Cassiopeia A (Aharonian et al. 2001) and the remnant of SN1006 (Mastichiadis \& de Jager 1996) Such emission is expected if nuclei are accelerated to $\mathrm{TeV}$ energies. They result from inelastic collisions between cosmic ray nuclei and nuclei in the ISM. These collisions produce (among other debris) neutral pions which decay into gamma rays.

Detailed models of the morphology and the spectrum of young SNRs seem to be the next step in order to get better tools to explain the observations. We consider the simplest model, where the X-ray emitting electrons are produced in the strong blastwave surrounding the remnant, and where the dominant emission mechanism for the X-ray continuum is synchrotron 
emission in the shock-compressed magnetic field. Other emission (and acceleration) mechanisms such as thermal or nonthermal Bremsstrahlung (e.g. Vink \& Laming 2003) are not considered here.

General considerations (e.g. Achterberg 2000) allow us to estimate the maximum attainable energy for shock-accelerated particles in a SNR as

$$
E_{\mathrm{max}} \approx Z e B\left(\frac{V_{\mathrm{s}}}{c}\right) R_{\mathrm{snr}}
$$

with $\mathrm{Ze}$ the absolute value of the particle charge, $B$ the magnetic field strength in the ISM, $V_{\mathrm{s}}$ the shock speed and $R_{\mathrm{snr}}$ the shock radius. This estimate applies to energetic nuclei, where radiation or ionization losses can be neglected. For typical parameters $\left(R_{\mathrm{snr}} \sim 10 \mathrm{pc}, B \sim 30 \mu \mathrm{G}\right.$ and $\left.V_{\mathrm{s}} \sim 1000 \mathrm{~km} \mathrm{~s}^{-1}\right)$ one finds $E_{\max } \sim 1000 \mathrm{TeV}$, intriguingly close to the energy of the knee.

Diffusive shock acceleration in the test-particle limit, where the back-reaction of the accelerated particles on the shock due the the associated pressure gradient is neglected, naturally produces particles with a power-law distribution in momentum. The power-law index $s$ is determined by the compression ratio $r$ of the shock. In the test-particle limit this slope equals $s=$ $(r+2) /(r-1)$, and spectral index of the associated optically thin synchrotron emission equals $\alpha \equiv-\mathrm{d} \ln S_{v} / \mathrm{d} \ln v=(s-1) / 2$. At radio frequencies observations of SNR shells give $\alpha \sim 0.5-0.6$, corresponding with $s=2 \alpha+1 \sim 2.0-2.2$. This is encouragingly close to the predicted value for $s$ if the accelerator is a strong hydrodynamical shock with compression ratio $r=4$. The fact that the observed spectrum is somewhat steeper than predicted probably indicates that the shock is weakened by the back-reaction of the accelerated particles, which reduces the shock compression (see e.g. Drury et al. 1989; Kang \& Jones 1991).

Even in the test-particle limit, the momentum distribution of the accelerated particles will deviate from a simple power law close to the maximum attainable energy. due to the combined effect of radiation losses and of the finite age of the remnant, the spectrum must steepen at high energy. Allen et al. (1999) show that the spectral index associated with five remnants (Cas A, Tycho, RCW 86, Kepler and SN 1006) must continue at the radio value $(\alpha \sim 0.6, s \sim 2.2)$ up to X-ray frequencies (photon energy $10 \mathrm{keV}$ ), corresponding to electron energies of $E \sim 10 \mathrm{TeV}$ for an assumed field strength of $10-100 \mu \mathrm{G}$. Above a photon energy of $10 \mathrm{keV}$ the photon spectral index is much steeper, $\alpha \sim 3.2$.

Reynolds (1998) (hereafter: R98) has presented a detailed calculation of the morphology and the spectrum of synchrotron $\mathrm{X}$-rays from SNRs. In this paper we present a method which expands on his work. We use a hydrodynamics code to calculate the evolution of a SNR in the general ISM. Simultaneously we calculate the electron acceleration at the shock, and electron transport within the remnant, including the effects of synchrotron losses and adiabatic losses in the post-shock flow and the inner regions of the remnant. This allows us to construct a detailed synchrotron spectrum of the remnant, showing the effects of the spectral roll-off at high frequencies due to the losses and finite age of the remnant. Our algorithm employs the test particle approximation where the fluid dynamics of the SNR is not influenced by the presence of the accelerated electrons (or nuclei, which we do not treat explicitly). We limit our calculations to electrons, and do not address the question of the acceleration of protons and other nuclei, and such nuclear processes as pion production and the associated gamma-ray emissivity of young supernova remnants.

This paper is organised as follows. In Sect. 2 we briefly describe the theory of particle acceleration at SNRs, and calculate typical electron- and photon energies. In Sect. 3 we describe the method used to simulate particle acceleration. In Sect. 4 we present the simulation results, followed by a discussion and conclusions in Sect. 5.

\section{Particle acceleration at SNR blastwaves}

\subsection{SNR evolution}

The evolution of a single supernova remnant (SNR) can be divided in four main stages (Woltjer 1972): the free expansion stage, the Sedov-Taylor stage, the pressure-driven snow plow stage and the momentum-conserving stage. These stages correspond to a progressive deceleration of SNR expansion. If the SNR expands into a uniform interstellar medium (ISM) with density $n_{0}$, the shock radius $R_{\mathrm{snr}}$ varies with time in these four stages as $R_{\mathrm{snr}} \propto t, R_{\mathrm{snr}} \propto t^{2 / 5}, R_{\mathrm{snr}} \propto t^{2 / 7}$ and $R_{\mathrm{snr}} \propto t^{1 / 4}$ respectively. In this paper we will only focus on the free expansion and Sedov-Taylor stages of SNR evolution. In late stages of SNR expansion, synchrotron losses together with the reduced efficiency of the acceleration process prevent electrons being accelerated to X-ray emitting energies.

To describe the transition between the free expansion stage and the Sedov-Taylor stage we use the results of McKee \& Truelove (1995). The transition occurs roughly when the SNR blastwave has swept up about 1.61 times the ejecta mass. For an interstellar mass density $\rho_{\text {ism }}$ this occurs at a radius

$R_{\mathrm{ST}}=1.17\left(\frac{3 M_{\mathrm{ej}}}{4 \pi \rho_{\mathrm{ism}}}\right)^{1 / 3}$,

which is

$R_{\mathrm{ST}}=2.23\left(\frac{M_{\mathrm{ej}}}{M_{\odot}}\right)^{1 / 3} n_{0}^{-1 / 3} \mathrm{pc}$.

Here $n_{0}=\rho_{\text {ism }} / \mu m$ is the number density (in $\mathrm{cm}^{-3}$ ) of the ISM, assuming a mean atomic mass $\mu_{\mathrm{m}}=2.34 \times 10^{-24} \mathrm{~g}$, and $M_{\mathrm{ej}}$ is the mass of the ejected stellar mantle. Given an mechanical explosion energy $E_{0}=10^{51} E_{51} \mathrm{erg}$, the transition from free expansion to the Sedov-Taylor stage occurs at an age

$t \approx t_{\mathrm{ST}}=209 E_{51}^{-1 / 2}\left(\frac{M_{\mathrm{ej}}}{M_{\odot}}\right)^{5 / 6} n_{0}^{-1 / 3} \mathrm{yr}$. 
Defining a typical velocity

$V_{\mathrm{ST}}=\frac{R_{\mathrm{ST}}}{t_{\mathrm{ST}}}=10400 E_{51}^{1 / 2}\left(\frac{M_{\mathrm{ej}}}{M_{\odot}}\right)^{-1 / 2} \mathrm{~km} \mathrm{~s}^{-1}$,

the typical shock speed at transition is

$V_{\mathrm{s}}\left(t_{\mathrm{ST}}\right) \approx 0.63 V_{\mathrm{ST}}=6552 E_{51}^{1 / 2}\left(\frac{M_{\mathrm{ej}}}{M_{\odot}}\right)^{-1 / 2} \mathrm{~km} \mathrm{~s}^{-1}$

\subsection{Shock acceleration}

The acceleration process around shocks relies on efficient scattering of the relativistic particles. This scattering is caused by hydromagnetic turbulence near the shock. The scattering confines particles near the shock and leads to repeated shock crossings by the particles resulting in acceleration due to the convergence of the up- and downstream "scattering centers" provided by the waves.

Spatial diffusion proceeds at a different rate along and across the magnetic field, and must be described by a diffusion tensor of the form

$\mathbf{D}_{\mathrm{x}}=\mathrm{D}_{\|} \hat{\boldsymbol{b}} \hat{\boldsymbol{b}}+\mathrm{D}_{\perp}(\mathbf{I}-\hat{\boldsymbol{b}} \hat{\boldsymbol{b}})$

where $\hat{\boldsymbol{b}}=\boldsymbol{B} /|\boldsymbol{B}|$ is the unit vector along the magnetic field and $\mathbf{I}$ the unit tensor. The field-aligned and perpendicular diffusion coefficients scale roughly as

$\mathrm{D}_{\|} \sim \frac{1}{3} c \ell=\kappa_{\mathrm{B}}\left(\frac{\ell}{r_{\mathrm{g}}}\right), \quad \mathrm{D}_{\perp} \sim \frac{\mathrm{D}_{\|}}{1+\left(\ell / r_{\mathrm{g}}\right)^{2}}$.

Here $\ell$ is the scattering mean free path, $r_{\mathrm{g}} \sim E / Z e B$ the particle gyration radius for relativistic particles $(E \approx \mathrm{pc})$ and

$\kappa_{\mathrm{B}} \equiv \frac{1}{3} c r_{\mathrm{g}}=\frac{c E}{3 Z e B}$

is the so-called Bohm diffusion coefficient.

In diffusive shock acceleration, the diffusion rate determines the acceleration time scale. The acceleration time near a planar shock equals (e.g. Drury 1983)

$t_{\mathrm{acc}} \equiv\left(\frac{1}{E} \frac{\mathrm{d} E}{\mathrm{~d} t}\right)^{-1}=\frac{3}{V_{1} V_{2}}\left(\frac{\mathrm{D}_{\mathrm{n} 1} V_{2}+\mathrm{D}_{\mathrm{n} 2} V_{1}}{V_{1}-V_{2}}\right)$.

Here $V$ is the velocity of the fluid in the shock rest frame, and $D_{n}$ denotes the diffusion coefficient in the direction perpendicular to the plane of the shock:

$\mathrm{D}_{\mathrm{n}} \equiv \hat{\boldsymbol{n}} \cdot \mathbf{D}_{\mathrm{x}} \cdot \hat{\boldsymbol{n}}=\mathrm{D}_{\|} \cos ^{2} \theta_{B n}+\mathrm{D}_{\perp} \sin ^{2} \theta_{B n}$.

Here $\hat{\boldsymbol{n}}$ is the shock normal and $\theta_{B n}$ the angle between the shock normal and the magnetic field. The subscripts 1 (2) refer to the values of various quantities upstream (downstream) of the shock.

In this paper we will assume Bohm diffusion, with $\ell \approx r_{\mathrm{g}}$ and $\mathrm{D}_{\|} \approx \mathrm{D}_{\perp} \approx \kappa_{\mathrm{B}}$. In this way we avoid having to deal with anisotropic diffusion, which is computationally expensive as one has to evaluate the local direction of the magnetic field at each time-step. Bohm diffusion is thought to be a reasonable approximation if the magnetic turbulence has an amplitude comparable to that of the mean field: $\delta B \sim B$. If such a turbulence level is present in the unshocked interstellar medium, shock compression of the magnetic field will not strongly change the value of $\delta B / B$.

Simulations (Lucek \& Bell 2000; Bell \& Lucek 2001) show that the interaction between cosmic rays streaming away from the shock and Alfvén waves leads to a rapid non-linear amplification of the upstream magnetic field. This leads to a much reduced cosmic ray mean free path, and, because of the high amplitude of the magnetic turbulence, $|\delta B| \sim 3-5 B$, to a moreor-less isotropic diffusion of the cosmic rays. This means that our results should apply approximately, with $B$ chosen to a few times the magnetic field strength in the undisturbed interstellar medium.

We estimate the acceleration time scale, assuming Bohm diffusion $\left(D_{\mathrm{n}} \approx D_{\mathrm{B}}\right)$ on both sides of a strong hydrodynamical shock. The shock has a compression ratio $r=4$ and propagates into a stationary ISM, so that $V_{1}=V_{\mathrm{s}}$ and $V_{2}=V_{\mathrm{s}} / 4$. Writing $B$ for the upstream magnetic field and $\kappa_{\mathrm{B}}$ for the associated Bohm diffusion coefficient, one finds

$t_{\mathrm{acc}}(E)=\xi \frac{\kappa_{\mathrm{B}}}{V_{\mathrm{s}}^{2}}=\frac{\xi}{3} \frac{c E}{Z e B V_{\mathrm{s}}^{2}}$.

The parameter $\xi$ takes the value $\xi=20$ for a parallel shock where the magnetic field is along the shock normal so that $B_{2}=$ $B$, and $\xi=8$ for a perpendicular shock where the field is in the plane of the shock and $B_{2}=4 B$. This corresponds to an acceleration rate for electrons or protons $(Z=1)$

$\left(\frac{\mathrm{d} E}{\mathrm{~d} t}\right)_{\mathrm{dsa}}=\frac{3}{\xi} \frac{e B V_{\mathrm{s}}^{2}}{c}$

The synchrotron loss rate for electrons is (e.g. Rybicki \& Lightman 1979)

$\left(\frac{\mathrm{d} E}{\mathrm{~d} t}\right)_{\mathrm{sy}}=-\frac{\sigma_{\mathrm{T}} B^{2} E^{2}}{6 \pi m_{\mathrm{e}}^{2} c^{3}}$

where $\sigma_{\mathrm{T}}=6.65 \times 10^{-25} \mathrm{~cm}^{2}$ is the Thomson cross section. Since we are only interested in an order-of-magnitude calculation, we neglect the difference between the synchrotron losses incurred upstream and downstream from the shock. This gives an approximate equation for the net electron acceleration rate at the shock, assuming Bohm diffusion:

$\frac{\mathrm{d} E}{\mathrm{~d} t}=\frac{3}{\xi} \frac{e B V_{\mathrm{s}}^{2}}{c}-\frac{\sigma_{\mathrm{T}} B^{2} E^{2}}{6 \pi m_{\mathrm{e}}^{2} c^{3}}$ 
Protons (and other nuclei) satisfy a similar equation without the synchrotron loss term. Equation (15) describes the energy gain of particles that remain close to the shock, subject to shock acceleration. One can use it to calculate the typical cut-off energy in the distribution of shock-accelerated particles.

\subsection{Typical electron and photon energies}

If losses can be neglected, as is the case for nuclei, Eq. (15) defines a typical energy

$E_{\max } \sim \frac{3 e B}{\xi}\left(\frac{V_{\mathrm{ST}}}{c}\right) R_{\mathrm{ST}}$,

which is

$$
\begin{aligned}
E_{\max } \approx & 200 E_{51}\left(\frac{\xi}{10}\right)^{-1}\left(\frac{M_{\mathrm{ej}}}{1 M_{\odot}}\right)^{-1 / 6} \\
& \times\left(\frac{B}{10 \mu \mathrm{G}}\right) n_{0}^{-1 / 3} \mathrm{TeV} .
\end{aligned}
$$

Particles injected into the acceleration process at a time $t \ll t_{\mathrm{ST}}$ will, in the absence of losses, reach an energy $E \approx E_{\max }$ at $t \sim t_{\mathrm{ST}}$. In the Sedov-Taylor phase the acceleration rate decays as $(\mathrm{d} E / \mathrm{d} t)_{\mathrm{dsa}} \propto V_{\mathrm{s}}^{2} \propto R_{\mathrm{snr}}^{-3}$. Assuming $B=$ constant, lossfree acceleration for $t>t_{\mathrm{ST}}$ typically triples the particle energy to $E \approx 3 E_{\max }$.

For electrons, the energy gain at the shock balances synchrotron losses when

$$
E=E_{\mathrm{sy}} \equiv \sqrt{\frac{27}{4 \xi}} \frac{m_{\mathrm{e}} c^{2}}{\sqrt{\alpha}}\left(\frac{B}{B_{\mathrm{cr}}}\right)^{-1 / 2}\left(\frac{V_{\mathrm{s}}}{c}\right)
$$

In this expression $\alpha \equiv e^{2} / \hbar c=1 / 137.04$ is the fine-structure constant, and $B_{\mathrm{cr}} \equiv m_{\mathrm{e}}^{2} c^{3} / e \hbar=4.4 \times 10^{13} \mathrm{G}$ is the critical magnetic field. For typical parameters one finds

$$
\begin{aligned}
E_{\mathrm{sy}} \approx & 350 E_{51}^{1 / 2}\left(\frac{\xi}{10}\right)^{-1 / 2}\left(\frac{V_{\mathrm{s}}}{V_{\mathrm{ST}}}\right)\left(\frac{M_{\mathrm{ej}}}{M_{\odot}}\right)^{-1 / 2} \\
& \times\left(\frac{B}{10 \mu \mathrm{G}}\right)^{-1 / 2} \mathrm{TeV} .
\end{aligned}
$$

In the free expansion stage one has $V_{\mathrm{s}} \approx 1.37 V_{\mathrm{ST}}$, while well into the Sedov-Taylor phase $\left(t \gg t_{\mathrm{ST}}\right)$ one has

$\frac{V_{\mathrm{s}}}{V_{\mathrm{ST}}} \approx 0.63\left(\frac{R_{\mathrm{snr}}}{R_{\mathrm{ST}}}\right)^{-3 / 2}$.

Asymptotically, electrons will approach an energy $E_{\text {sy }}$ when the age of the remnant becomes larger than the synchrotron loss time,

$t_{\mathrm{sy}} \approx 800\left(\frac{B}{10 \mu \mathrm{G}}\right)^{-2}\left(\frac{E}{100 \mathrm{TeV}}\right)^{-1} \mathrm{yr}$.

The scaling $E_{\text {sy }} \propto B^{-1 / 2}$ implies that the maximum energy of synchrotron photons no longer depends on the magnetic field strength in the source once electron acceleration is limited by synchrotron losses. Using the standard expression for the typical synchrotron frequency $v_{\mathrm{s}}$,

$v_{\mathrm{s}} \sim \frac{3 e B}{4 \pi m_{\mathrm{e}} c}\left(\frac{E}{m_{\mathrm{e}} c^{2}}\right)^{2}$

with $E=E_{\text {sy }}$, one finds:

$h v_{\max } \sim\left(\frac{81}{4 \pi \xi \alpha}\right) m_{\mathrm{e}} V_{\mathrm{s}}^{2}$

which is:

$h v_{\max } \approx 54 E_{51}\left(\frac{\xi}{10}\right)^{-1}\left(\frac{M_{\mathrm{ej}}}{M_{\odot}}\right)^{-1}\left(\frac{V_{\mathrm{s}}}{V_{\mathrm{ST}}}\right)^{2} \mathrm{keV}$.

Up to factors of order unity, one has (McKee \& Truelove 1995)

$V_{\mathrm{ST}}^{2}=2 E_{0} / M_{\mathrm{ej}}$,

so that the typical cut-off energy in the spectrum of synchrotron photons is

$h v_{\max } \approx 180\left(\frac{\xi}{10}\right)^{-1}\left(\frac{m_{\mathrm{e}}}{M_{\mathrm{ej}}}\right)\left(\frac{V_{\mathrm{s}}}{V_{\mathrm{ST}}}\right)^{2} E_{0}$,

which depends in a simple manner on the total explosion energy and the ejecta mass, as $V_{\mathrm{s}} \approx V_{\mathrm{ST}}$ when the maximumenergy particles are produced. These estimates show that one has to observe SNRs in the free expansion phase (where $V_{\mathrm{s}} \approx$ $V_{\mathrm{ST}} \approx 10000 \mathrm{~km} \mathrm{~s}^{-1}$ ), or closely thereafter, if the shock is to accelerate electrons to a sufficiently high energy to produce synchrotron $\mathrm{X}$-rays in the $\mathrm{keV}$ range.

Figure 1 shows the typical energy of electrons and protons, injected at an early stage of the SNR evolution, as a function of time. The particle energy is calculated by numerically integrating Eq. (15) with $\xi=20$ (parallel shock), using the semianalytical results of McKee \& Truelove (1995) for the velocity of the external blast wave as a function of time:

$\frac{V_{\mathrm{S}}(t)}{V_{\mathrm{ST}}}= \begin{cases}1.37\left[1+0.6\left(\frac{t}{t_{\mathrm{ST}}}\right)^{3 / 2}\right]^{-5 / 3} & \text { if } t<t_{\mathrm{ST}}, \\ 0.63\left[1.56\left(\frac{t}{t_{\mathrm{ST}}}\right)-0.56\right]^{-3 / 5} & \text { if } t \geq t_{\mathrm{ST}} .\end{cases}$

The parameters for the SNR are the same as used in the simulations presented below: an explosion energy $E_{0}=10^{51} \mathrm{erg}$, ejecta mass $M_{\mathrm{ej}}=3 M_{\odot}$ and an ISM mass density $\rho_{0}=$ $10^{-24} \mathrm{~g} / \mathrm{cm}^{3}$. This corresponds to $t_{\mathrm{ST}} \approx 700 \mathrm{yr}, V_{\mathrm{ST}} \approx$ $6000 \mathrm{~km} \mathrm{~s}^{-1}$ and $R_{\mathrm{ST}} \approx 4.5 \mathrm{pc}$. We consider three values for the strength of the ISM magnetic field: $B_{0}=3,10$ and $30 \mu \mathrm{G}$.

In the free expansion stage $\left(t \ll t_{\mathrm{ST}}\right.$ and $\left.R_{\mathrm{snr}} \ll R_{\mathrm{ST}}\right)$ the energy of the accelerated particles (nucleons and electrons) is 

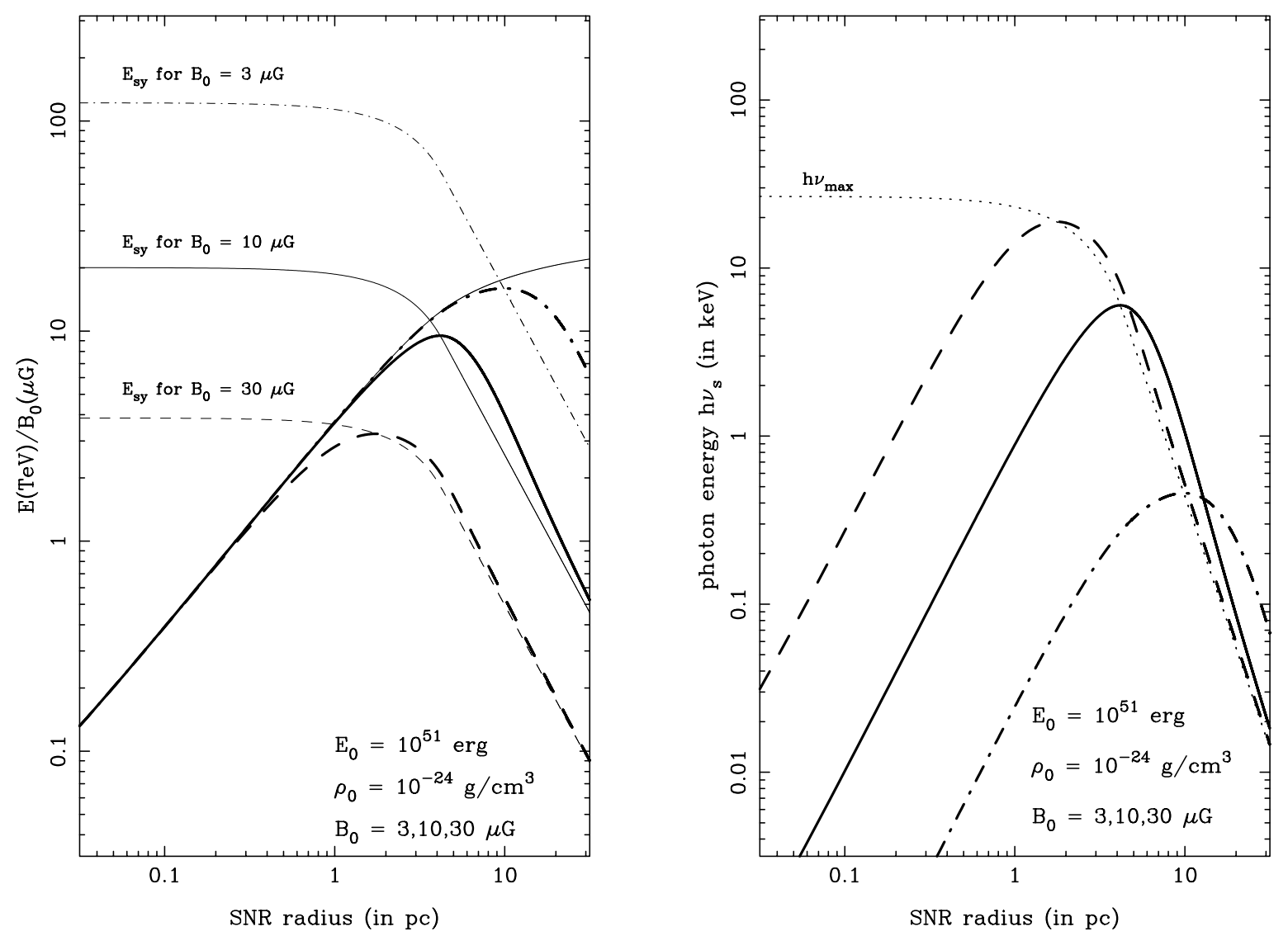

Fig. 1. The left panel shows the typical maximum energy of shock-accelerated electrons and protons, plotted as $E / B$, as a function of the radius $R_{\text {snr }}$ of the supernova remnant. Shown are three cases, corresponding to a magnetic field strength of $B=3 \mu \mathrm{G}$ (dash-dot curves), $B=10 \mu \mathrm{G}$ (solid curves) and $B=30 \mu \mathrm{G}$ (dashed curves). Also shown is the energy $E_{\text {sy }}$ where electron synchrotron losses balance the energy gain per unit time at the shock. At early times, the maximum particle energy rises as $E / B \propto V_{\mathrm{snr}} R_{\mathrm{snr}}$. Electrons start to loose energy when $E>E_{\text {sy }}$, asymptotically approaching the relation $E \approx E_{\mathrm{sy}} \propto R_{\mathrm{snr}}^{-3 / 2}$ at late times. Protons, who are not subject to losses, continue to follow the loss-free energy curve (thin solid curve). The right panel shows the typical maximum energy of synchrotron photons emitted by shockaccelerated electrons in these three cases. Also shown is the energy $h v_{\max }$ corresponding to an electron energy $E=E_{\text {sy }}$ (dotted curve). This quantity is independent of the magnetic field strength. In older remnants, the cut-off in the synchrotron spectrum should be close to a photon energy $h v_{\max }$.

limited by the finite age of the source. The maximum particle energy grows as $E \propto B R_{\mathrm{snr}}$. Proton acceleration continues to be limited only by the size and age of the remnant, and protons are accelerated to an energy $E \approx 20 B_{\mu \mathrm{G}} \mathrm{TeV}$ by the time the SNR has expanded to a radius of $30 \mathrm{pc}$.

Electrons feel the effect of synchrotron losses. The influence of these losses sets in at earlier times (and smaller SNR radii) with increasing field strength. This limits the electron energy to $E \leq 40 \mathrm{TeV}$ for $B_{0}=3 \mu \mathrm{G}, E \leq 90 \mathrm{TeV}$ for $B_{0}=10 \mu \mathrm{G}$ and $E \leq 100 \mathrm{TeV}$ for $B_{0}=30 \mu \mathrm{G}$. At late times (large radii) the energy of old electrons near the shock decays to $E=E_{\mathrm{sy}}$. This means that the maximum energy in the distribution of shock-accelerated electrons will correspond to the energy where shock acceleration is balanced by synchrotron losses.

Figure 1 also shows the typical photon energy $h v_{\mathrm{s}}$ of the synchrotron photons emitted by these maximum-energy electrons. It gives the typical cut-off energy in the photon spectrum.
If the magnetic field is small, electrons hit the synchrotronlimited energy relatively late in the evolution of the remnant $\left(t \gg t_{\mathrm{ST}}\right)$, when the associated maximum photon energy is already decaying: $h v_{\max } \propto V_{\mathrm{s}}^{2} \propto R_{\mathrm{snr}}^{-3}$. Such remnants are not expected to be strong sources of non-thermal X-ray emission.

If the magnetic field is large, shock acceleration is rapid, and the electrons hit the synchrotron-limited energy early in the evolution of the remnant. In that case, the remnant is still in the free expansion stage with $V_{\mathrm{s}} \approx V_{\mathrm{ST}}$.

Taking $\xi \approx 15$ as an average value, such remnants should show non-thermal X-ray emission around $t \approx t_{\mathrm{ST}}$, up to typical photon energies of the order of $10-100 \mathrm{keV}$.

One can estimate a minimum value of the magnetic field above which one expects young SNRs to be non-thermal X-ray emitters. It corresponds to the case where the electrons begin to be limited by synchrotron losses at the end of the freeexpansion stage, so that $E_{\mathrm{max}} \approx E_{\mathrm{sy}}$ for $R_{\mathrm{snr}} \approx R_{\mathrm{ST}}$. It turns out 
that this minimum field only depends on the SNR parameters through the Sedov-Taylor radius $R_{\mathrm{ST}}$. It can be expressed as:

$\frac{B_{\mathrm{min}}}{B_{\mathrm{cr}}}=\left(\frac{3 \xi}{4 \alpha}\right)^{1 / 3}\left(\frac{\lambda_{\mathrm{e}}}{R_{\mathrm{ST}}}\right)^{2 / 3}$

where $\lambda_{\mathrm{e}}=\hbar / m_{\mathrm{e}} c=3.86 \times 10^{-11} \mathrm{~cm}$ is the Compton wavelength of the electron. For typical SNR parameters one finds

$B_{\min } \approx 14\left(\frac{\xi}{10}\right)^{1 / 3}\left(\frac{M_{\mathrm{ej}}}{M_{\odot}}\right)^{-2 / 9} n_{0}^{2 / 9} \mu \mathrm{G}$.

The value of $B_{\text {min }}$ depends relatively weakly on SNR parameters.

\section{Simulation method}

\subsection{SNR hydrodynamics}

We have performed hydrodynamical simulations, using the Versatile Advection Code ${ }^{1}$ (VAC, Tóth 1996). We employ the TVD-MUSCL scheme with a Roe-type approximate Riemann solver, one of the numerical algorithms available in VAC (Tóth \& Odstrčil 1996). A discussion of this (and other) schemes for numerical hydrodynamics can be found in LeVeque (1998).

The calculations are performed in one dimension, on a spherically symmetric grid with uniform radial grid spacing. As an initial condition we deposit an amount $E_{0}$ in thermal energy in a mass $M_{\text {ej }}$ in the first few grid cells. This leads to the formation of both the reverse shock propagating into the stellar ejecta, and the forward shock (blast wave) in the ISM, as discussed above. The resolution of our calculations are such that both the forward shock and the reverse shock are resolved, and these shocks have the correct compression factor $(r=4)$ expected for a strong, non-relativistic hydrodynamical shock.

\subsection{Magnetic fields}

Our hydrodynamical simulations do not include the dynamical influence of magnetic fields. The magnetic pressure $\left(B^{2} / 8 \pi\right)$ is generally much lower than the thermal pressure inside a young SNR. Therefore, the presence of magnetic fields does not significantly influence SNR expansion. However, in order to describe particle acceleration and the associated synchrotron emission, we need to know the value for the magnetic field throughout the SNR. The magnetic field determines the value of the particle diffusion coefficient, and of the synchrotron emissivity.

We choose to describe the magnetic field in the approximation where it is passively carried by the flow, but neglect the magnetic forces acting on the flow. The magnetic field configuration inside the SNR can then be found using the frozen-in condition (flux conservation). This condition allows us to calculate the magnetic field $\boldsymbol{B}(\boldsymbol{r}, t)$, given the field $\boldsymbol{B}_{0}\left(\boldsymbol{r}_{0}, t_{0}\right)$ and local density $\rho_{0}$ at some initial time $t_{0}$ when the fluid attached

\footnotetext{
${ }^{1}$ See http://www.phys.uu.nl/ ${ }^{\sim}$ toth/
}

to the field line was at position $\boldsymbol{r}\left(t_{0}\right) \equiv \boldsymbol{r}_{0}=\left(x_{0}^{1}, x_{0}^{2}, x_{0}^{3}\right)$ (e.g. Zeldovich et al. 1983). The field components $B_{i}$ follow quite generally from

$\frac{B_{i}}{\rho h_{i}}=\sum_{j=1}^{3} \frac{\partial x^{i}}{\partial x_{0}^{j}}\left(\frac{B_{0 j}}{\rho_{0} h_{0 j}}\right)$.

Here $\rho \equiv \rho(r, t)$ is the density and $\rho_{0} \equiv \rho\left(r_{0}, t_{0}\right)$ etc. The coefficients $h_{i}$ are the scale factors appearing in the distance recipe for orthogonal curvilinear coordinates $x^{i}(i=1,2,3)$, $\mathrm{d} s^{2}=\sum_{i=1}^{3}\left(h_{i} \mathrm{~d} x^{i}\right)^{2}$. In spherical coordinates, with distance recipe

$\mathrm{d} s^{2}=\mathrm{d} r^{2}+r^{2} \mathrm{~d} \theta^{2}+r^{2} \sin ^{2} \theta \mathrm{d} \phi^{2}$,

one has $h_{r r}=1, h_{\theta \theta}=r^{2}$ and $h_{\phi \phi}=r^{2} \sin ^{2} \theta$. The assumption of radial expansion, together with mass conservation

$4 \pi r^{2} \rho \mathrm{d} r=4 \pi r_{0}^{2} \rho_{0} \mathrm{~d} r_{0}$,

implies:

$\frac{\partial \theta}{\partial r_{0}}=\frac{\partial r}{\partial \theta_{0}}=0, \quad \frac{\partial \theta}{\partial \theta_{0}}=1, \quad \frac{\partial r}{\partial r_{0}}=\frac{\rho_{0} r_{0}^{2}}{\rho r^{2}}$.

Assuming $B_{\phi}=0$, one finds the radial and tangential field components $B_{r}$ and $B_{\theta}$ :

$B_{r}=\left(\frac{r_{0}}{r}\right)^{2} B_{0 r}$

$B_{\theta}=\left(\frac{\rho}{\rho_{0}}\right)\left(\frac{r}{r_{0}}\right) B_{0 \theta}$.

Here $r$ is the current radial position of the fluid element, and $r_{0}$ its position when it crosses the SNR blastwave. Similarly, $\rho$ is the density at the current position of the fluid element and $\rho_{0}$ is the density of the ISM. We assume that the only magnetic field present in the SNR is the compressed interstellar field. The interstellar field is taken to be uniform with field strength $B_{0}$ and aligned with the $z$-axis:

$B_{0 r}=B_{0} \sin \theta_{0}, \quad B_{0 \theta}=-B_{0} \cos \theta_{0}$.

The density profile $\rho(r)$ and the value of $r / r_{0}$ throughout the remnant follow from the simulations. In this manner we obtain a remnant with a spherically symmetric flow, and an axially symmetric magnetic field configuration, which both dependent on time. This approach is similar to the one used Duin \& Strom (1975) and by Reynolds \& Chevalier (1981).

This approach neglects the contribution of the turbulent component of the magnetic field. In order to model this one would have to add such a component, for instance by modelling turbulence on a wide range of scales, and calculate its interaction with the shock. This is beyond the scope of the present paper. This will not strongly affect the results obtained below for 
the total synchrotron emissivity as long as $\delta B \leq B$. Obviously, a strong turbulent field component would change the degree of polarization of the radiation which, at least for X-rays, is an unobservable quantity.

The difference between our approach and the approach taken in R98 is in the treatment of the dynamical evolution of the SNR. Whereas R98 assumes a Sedov solution, our numerical results cover the free expansion stage, the Sedov-Taylor stage, and the transition between the two. As argued above, the most energetic particles are produced near this transition.

\subsection{Energetic particles}

The acceleration and propagation of relativistic particles in a magnetized astrophysical plasmas is usually investigated by solving a Fokker-Planck equation of the type (e.g. Skilling 1975; Jones 1990):

$$
\frac{\partial F(\boldsymbol{Z}, t)}{\partial t}+\frac{\partial}{\partial \boldsymbol{Z}} \cdot \boldsymbol{S}(\boldsymbol{Z}, t)=Q(\boldsymbol{Z}, t)
$$

Here $F(\boldsymbol{Z}, t)$ is the particle distribution function in phase space, $\boldsymbol{Z}=(\boldsymbol{x}, \boldsymbol{p})$ is the phase-space position vector,

$\boldsymbol{S}(\boldsymbol{Z}, t)=\left(\frac{\mathrm{d} \boldsymbol{Z}}{\mathrm{d} t}\right) F(\boldsymbol{Z}, t)-\mathbf{D} \cdot \frac{\partial F(\boldsymbol{Z}, t)}{\partial \boldsymbol{Z}}$

is the flux in phase space, which consists of an advective and a diffusive part with $\mathrm{d} \boldsymbol{Z} / \mathrm{d} t$ the mean phase-space advection velocity and $\mathbf{D}$ a diffusion tensor, formally defined in dyadic notation as

$\mathbf{D} \equiv \frac{\langle\Delta \boldsymbol{Z} \Delta \boldsymbol{Z}\rangle}{2 \Delta t}$

The term $Q(\boldsymbol{Z}, t)$ describes the injection of fresh particles into the acceleration process.

It has been shown (e.g. Gardiner 1983; Saslaw 1985) that the Fokker-Planck equation is equivalent to the following stochastic differential equation (SDE) of the Itô form, consisting of a regular advective term $\propto \mathrm{d} t$ and a stochastic term:

$\mathrm{d} \boldsymbol{Z}=\dot{\boldsymbol{Z}} \mathrm{d} t+\sqrt{2 \mathbf{D}} \cdot \mathrm{d} \boldsymbol{W}$.

Here $\sqrt{\mathbf{D}}$ is short-hand notation for a tensor with components such that

$$
\sqrt{\mathbf{D}} \cdot \sqrt{\mathbf{D}}=\mathbf{D} \text {. }
$$

The velocity $\dot{\boldsymbol{Z}}$ entering the advective term consists of both the mean velocity in phase space, and an additional dynamical friction term:

$$
\dot{\boldsymbol{Z}} \equiv \frac{\mathrm{d} \boldsymbol{Z}}{\mathrm{d} t}+\frac{\partial}{\partial \boldsymbol{Z}} \cdot\left(\mathbf{D}^{\dagger}\right)
$$

where $\mathbf{D}^{\dagger}$ is the transpose of $\mathbf{D}$.

The noise term $d \boldsymbol{W}$ in the stochastic term of Eq. (39) is a $N$-dimensional Wiener process, where $N$ is the number of degrees of freedom in phase space (e.g. MacKinnon \& Craig 1991; Achterberg \& Krülls 1992; Krülls \& Achterberg 1994). Its components $\mathrm{d} W_{i}$ satisfy a set of simple rules,

$\left\langle\mathrm{d} W_{i}\right\rangle=0, \quad\left\langle\mathrm{~d} W_{i} \mathrm{~d} W_{j}\right\rangle= \begin{cases}\mathrm{d} t & \text { if } i=j \\ 0 & \text { if } i \neq j,\end{cases}$

where the angular brackets indicate an average over many statistically independent realizations of this Wiener process.

By running many independent realizations ("particle orbits") of prescription (39) one can construct the phase-space distribution $F(\boldsymbol{Z}, t)$. Injection of fresh particles is straightforward: one simply introduces new particles to the pool of particles which follow the above prescription.

This correspondence between the Fokker Planck equation and SDEs is the basis for for a fast simulation method for particle acceleration and propagation in a time-dependent flow. With this method one can include effects like synchrotron losses, Compton losses and expansion losses in a straightforward fashion.

The application to shock acceleration has been considered by different authors (e.g Krülls \& Achterberg 1994; Marcowith \& Kirk 1999) and has been compared succesfully with available analytical solutions. We employ this method here in conjunction with the hydrodynamical simulations to calculate the behaviour of relativistic electrons a young supernova remnant. Casse \& Marcowith (2003) have applied this method to propagate particles through a static magnetised jet.

For practical reasons, we assume isotropic spatial diffusion with a diffusion coefficient equal to the Bohm diffusion coefficient, $\mathbf{D}_{\boldsymbol{x}}=\kappa_{\mathrm{B}} \mathbf{I}$, and neglect momentum diffusion (secondorder Fermi acceleration) due to the hydromagnetic waves. Spatial transport is then described in cylindrical coordinates $Z=r \cos \theta$ and $R=r \sin \theta$ by the following two equations:

$$
\begin{aligned}
& \mathrm{d} Z=\left(V \cos \theta+\frac{\partial \kappa_{\mathrm{B}}}{\partial Z}\right) \mathrm{d} t+\sqrt{2 \kappa_{\mathrm{B}}} \mathrm{d} W_{Z} \\
& \mathrm{~d} R=\left(V \sin \theta+\frac{\partial \kappa_{\mathrm{B}}}{\partial R}\right) \mathrm{d} t+\sqrt{2 \kappa_{\mathrm{B}}} \mathrm{d} W_{R} .
\end{aligned}
$$

Here $V$ is the radial fluid velocity at the position of the particle. We neglect the possible influence of Fermi-II acceleration by waves, so the particle momentum $p$ does not diffuse. In that case the energy change for relativistic electrons with $E \approx \mathrm{pc}$ is described by (e.g. Krülls \& Achterberg 1994):

$\mathrm{d} E=-\frac{E}{3}\left[\frac{1}{r^{2}} \frac{\partial}{\partial r}\left(r^{2} V\right)\right] \mathrm{d} t+\left(\frac{\mathrm{d} E}{\mathrm{~d} t}\right)_{\mathrm{sy}} \mathrm{d} t$.

The first term, involving the divergence of the radial fluid velocity, describes expansion losses within the remnant, as well as shock acceleration at the forward and reverse shocks, provided the time step $\mathrm{d} t$ is chosen such that (e.g. Krülls \& Achterberg 1994)

$V \mathrm{~d} t \ll \Delta r_{\mathrm{s}} \ll \sqrt{2 \kappa_{\mathrm{B}} \mathrm{d} t}$ 


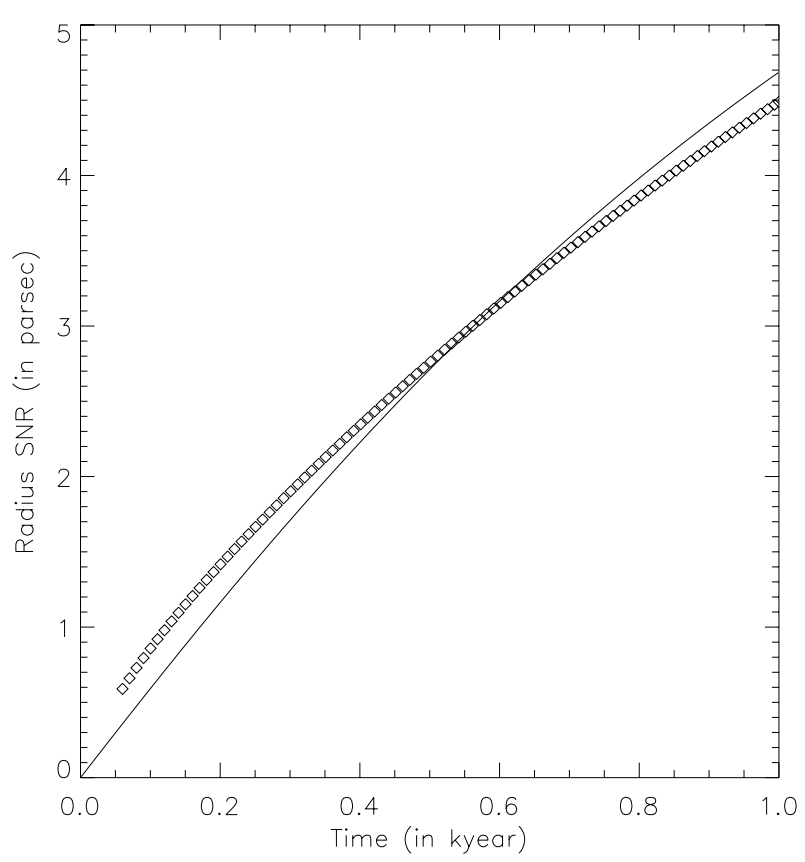

Fig. 2. Expansion of a SNR, as calculated with the hydro-code (diamonds), and the semi-analytical result of McKee \& Truelove (solid line). The semi-analytical result slightly underestimates (overestimates) the supernova remnant radius for times $t<0.5 \mathrm{kyr}(t>$ $0.5 \mathrm{kyr})$. McKee \& Truelove (1995) find deviations of a similar magnitude. Such small differences probably result from the different ways in which the initial explosion is modelled numerically. The parameters of the SNR are tabulated in Table 1.

The quantity $\Delta r_{\mathrm{s}}$ is the thickness of the shock transition, typically a few grid cells. In the simulations, the shocks are thin regions of strong (negative) divergence. The second term in (43) is the synchrotron loss term (14), evaluated using the local value of the magnetic field. Even though Eq. (43) has no explicit stochastic term, the energy gain exhibits stochastic behaviour because different realizations of an electron trajectory through the remnant, as obtained from (42), lead to a different sampling of the fluid velocity $V(r, t)$ and its derivatives.

By simultaneously advancing the particle position $(R, Z)$ in the remnant and the particle energy $E$ using (42) and (43), using the velocity $V(r, t)$ obtained from the numerical simulations, one can model the behaviour of energetic electrons in the SNR.

\section{Simulation results}

We have simulated the evolution of a spherical supernova remnant with the parameters listed in Table 1 using the VAC code. Figure 2 shows the radius $R_{\text {snr }}$ of the remnant as a function of time, comparing a numerical simulation with the semianalytical results of McKee \& Truelove (1995)

Figure 3 shows the radial velocity profile $V(r)$ of the remnant at a moment close to the transition to the Sedov-Taylor phase. One can clearly identify the reverse shock propagating into the ejecta ( $r \simeq 3.6$ parsec), and the forward shock ( $r \simeq 4.5$ parsec) propagating into the interstellar medium.

The results from the hydrodynamical simulations are used to calculate the acceleration and propagation of relativistic
Table 1. Parameters of simulated supernova remnant.

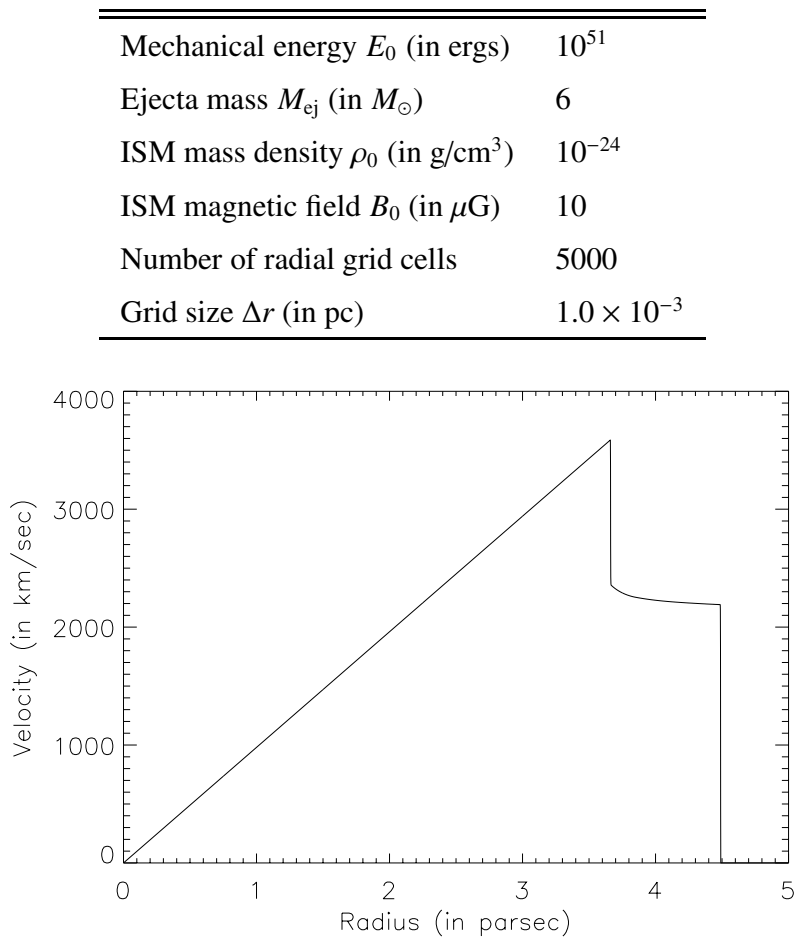

Fig. 3. Velocity profile of a SNR with the parameters as in Table 1.

electrons near the forward shock (and some additional acceleration at the reverse shock). in the test-particle approximation, using the Itô method described above. We assume isotropic diffusion at the Bohm rate set by the magnetic field constructed using the flux-freezing condition.

We continuously inject particles at the forward shock, starting at the age of $t=200$ years, up to the end of the simulation at an age of $t=1000$ years. At the end of the simulation the radius of the SNR equals $R_{\mathrm{snr}} \simeq 4.5$ parsec. The total grid over which the test-particles are allowed to propagate has a size $0.0 \leq R \leq 6.0$ parsec in cylindrical radius, and $-6.0 \leq Z \leq+6.0$ parsec along the symmetry axis (Z-axis). A total of $\sim 4.2 \times 10^{6}$ test particles are injected during the simulation. The injection is at constant injection momentum $p_{0}$, with an injection rate proportional to the amount of interstellar material swept-up by the forward shock per unit time. The number of particles introduced at the shock in a time interval $\mathrm{d} t$ scales as

$\mathrm{d} \mathcal{N}(p) \propto R_{\mathrm{snr}}^{2} V_{\mathrm{s}} \mathrm{d} t \times \delta\left(p-p_{0}\right)$.

We keep the injection momentum $p_{0}$ fixed during the simulation. One might argue that the injection momentum is proportional to the shock speed, corresponding to a few times the typical "thermal" particle momentum in the post-shock flow. However, during the simulation the outer shock decelerates from the initial expansion velocity of $\sim 5300 \mathrm{~km} \mathrm{~s}^{-1}$ to $\sim 2200 \mathrm{~km} \mathrm{~s}^{-1}$, so the post-shock (thermalised) momentum only varies by a factor $\sim 2$. 
This implies that most particles were injected at later times, with $\mathrm{d} \mathcal{N} \propto t^{2} \mathrm{~d} t$ in the free expansion phase, and $\mathrm{d} \mathcal{N} \propto t^{1 / 5} \mathrm{~d} t$ in the Sedov-Taylor stage.

At the end of the simulation we have the position and the momentum of each simulated particle. Because we also know the magnetic field strength throughout the remnant we can produce synchrotron surface brightness maps at different frequencies. The emissivity of each particle is modelled using a tabulated form of the well-known synchrotron emission profile $F\left(v / v_{\mathrm{s}}\right)($ e.g. Rybicky \& Lightman 1979)

$P(v)=\frac{\sqrt{3} e^{3} B \sin \alpha}{m_{\mathrm{e}} c^{2}} F\left(\frac{v}{v_{\mathrm{s}}}\right)$,

where the typical frequency $v_{\mathrm{s}}$ has been defined in Eq. (22). To produce a surface brightness map we exploit the cylindrical symmetry of the system. A test-particle is copied $N$ times on a circle around the axis of symmetry. In this way, each simulated electron contributes $N$ times to the synchrotron map, each time at a different position separated by an angle $2 \pi / N$ from the previous copy. Each copy has a different angle $\alpha$ between the line of sight and the magnetic field orientation, which determines the typical emissivity and frequency distribution of the synchrotron radiation (see Eqs. (22) and (46)). By adding the contribution of all copy particles one produces a surface brightness map.

An example of such a synchrotron map is shown in Fig. 4. It shows a similar surface brightness distribution as the synchrotron maps presented by R98.

Figure 5 shows the energy distribution of all the testparticles in the SNR. One can see that the cut-off occurs at the expected energy of $E \simeq 100 \mathrm{TeV}$.

One can also calculate the integrated photon spectrum due to synchrotron radiation from all the simulated electrons in the remnant, using once again the trick of copy-particles with different values of $\alpha$. Figure 6 shows the resulting integrated synchrotron spectrum.

At low photon energies, the spectral index $\alpha$ equals $\alpha \simeq$ -1.0 , whereas at the roll-off part of the spectrum, where the synchrotron losses start to match the energy gain due to the acceleration process we get a spectral index of $S_{v} \propto v^{-3.0}$ corresponding with a spectral index for the momentum distribution of $s=7.0$. This is encouraging close to the value as observed for the five remnants by Allen et al. (1999).

\section{Discussion and conclusions}

We have considered the acceleration of electrons in a young SNR by combining a simple hydrodynamics calculation with an algorithm to describe electron acceleration in the test-particle limit. These calculations, as well as theoretical estimates based on a simple model of shock acceleration, confirm that young remnants can accelerate electrons up to energies of $10-100 \mathrm{TeV}$, provided the magnetic field is sufficiently strong, typically $B \geq 10 \mu \mathrm{G}$. We predict that these remnants are sources of synchrotron X-rays around the transition time between the free expansion phase and the Sedov-Taylor phase.

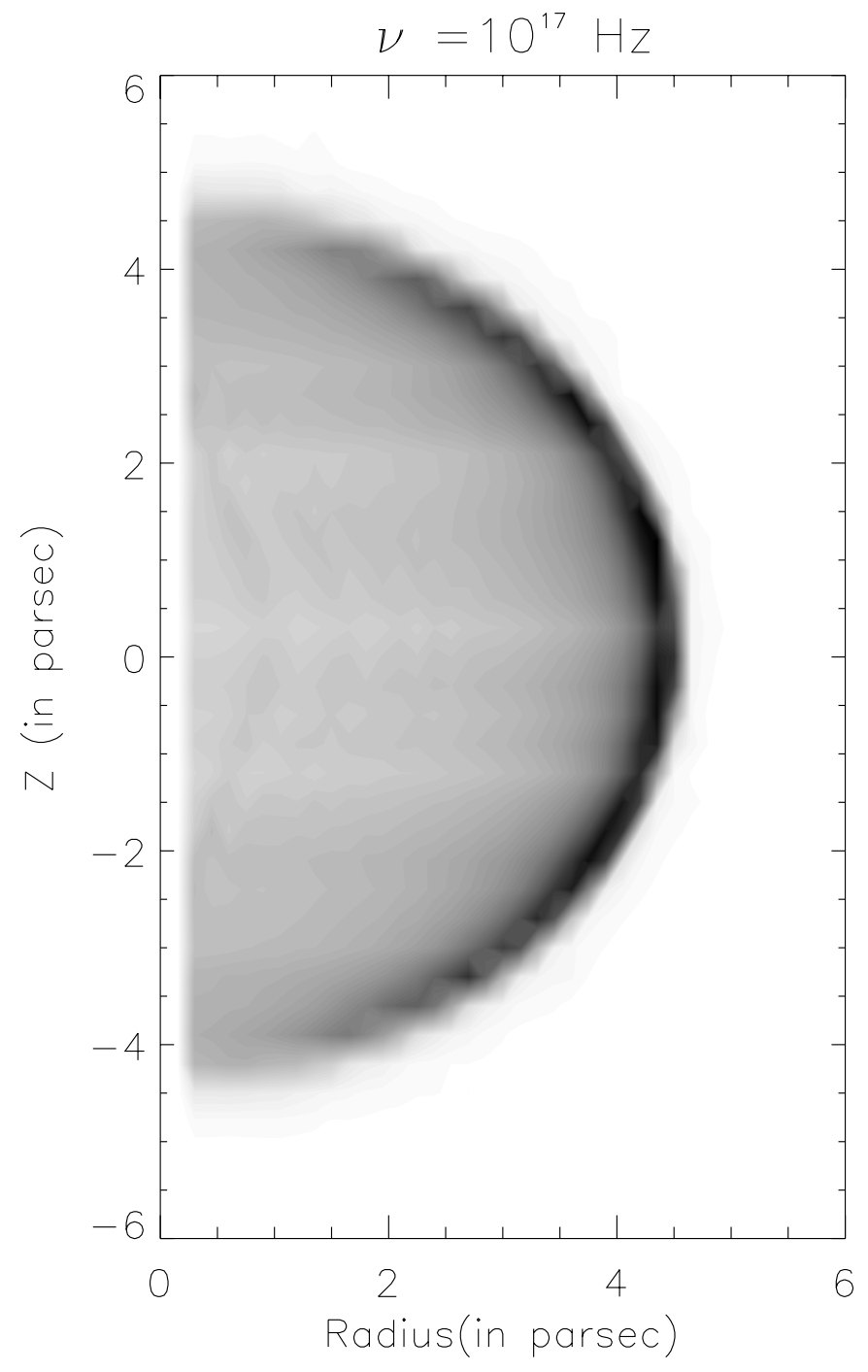

Fig. 4. Synchrotron map at a frequency of $v=10^{17} \mathrm{~Hz}$, as viewed by an observer located in a plane perpendicular to the undisturbed interstellar magnetic field at the explosion site.

We have produced a synchrotron surface brightness map at $h v \sim 1 \mathrm{keV}$ which is qualitatively comparable with the synchrotron maps produced by R98, who assumed a SedovTaylor remnant. The spectra we produce have a spectral in$\operatorname{dex} \mathrm{d} \ln S_{v} / \mathrm{d} \ln v \sim-2$ at $h v \geq 10 \mathrm{keV}$, close to the value observed for the non-thermal X-ray emission in several young supernova remnants.

It should be pointed out that there is the possibility that $\mathrm{X}$-ray continuum emission is -at least in part- generated by bremsstrahlung in a hot electron gas which derives this energy from another acceleration process, not directly associated with the outer blast wave, but with plasma shocks within the remnant. Such a model was proposed by Vink \& Laming (2003) in their detailed analysis of the remnant Cassiopeia A. In this remnant, they estimate a high magnetic field strength, $B \sim 0.08-0.16 \mathrm{mG}$, much stronger than the field strength assumed in this paper. Our model obviously does not apply to this situation. 


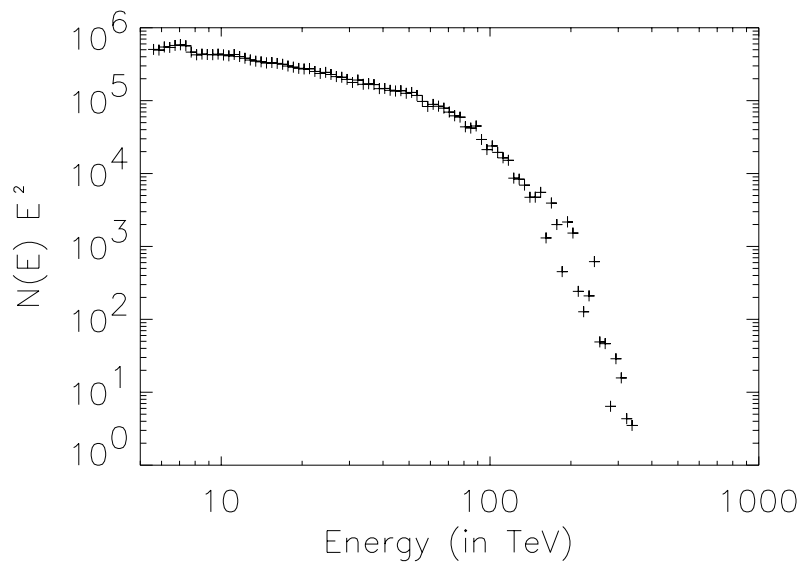

Fig. 5. Energy distribution of the total amount of test-particles in the SNR.

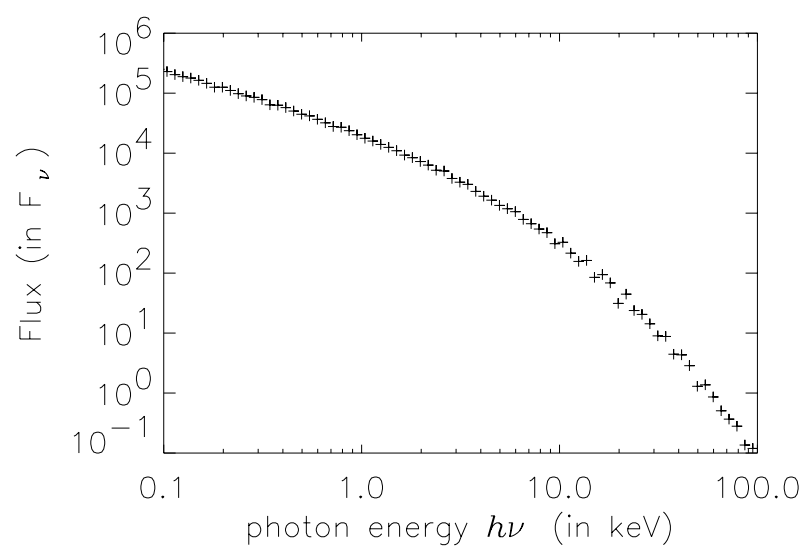

Fig. 6. Total integrated spectrum of the SNR.

Our method can be applied to more complicated problems, such as SNRs expanding into a non-uniform ISM, which will lead to a more complicated morphology. Also, some of the simplifying assumptions made in these calculations should be replaced by more realistic ones. First of all, particle diffusion in the remnant should be treated more generally, allowing for the anisotropy induced by the magnetic field by relaxing the assumption of Bohm diffusion where the mean free path equals the gyration radius $\left(\ell \sim r_{\mathrm{g}}\right)$. Although anisotropic diffusion is easily incorporated into our formalism, it is computationally expensive. We expect that, at least in the limit $\ell \gg r_{\mathrm{g}}$, this will introduce a larger difference between the particle acceleration at the quasi-parallel region of the blastwave (where $\theta_{B n}<45^{\circ}$ ) and the quasi-perpendicular region (where $\theta_{B n}>45^{\circ}$ ).

Secondly, one could allow for distributed acceleration by waves (Fermi-II acceleration) in the remnant by assuming some wave spectrum, and including the associated momentum diffusion into the equation governing the energy change of our test particles.

Finally, one would like to relax the assumptions of spherical symmetry and a uniform interstellar medium, using a full MHD treatment to calculate both the magnetic field in the SNR, and the small-scale structure caused by instabilities (such as the Rayleigh-Taylor instability) expected in an expanding SNR.
Acknowledgements. The authors would like to thank Yves Gallant and Luke Drury for discussions, and the referee, Dr. A Marcowith, for a number of valuable comments.

\section{References}

Achterberg, A. 2000, in Highly Energetic Physical Processes and Mechanisms for Emission from Astrophysical Plasmas, IAU Symp. 195, ed. P. C. H. Martens, S. Tsuruta, \& M. A. Weber, ASP, 291

Achterberg, A., \& Krülls, W. M. 1992, A\&A, 265, L13

Aharonian, F., Akhperjanian, A., Barrio, J., et al. 2001, A\&A, 370, 112

Allen, G. E., Gotthelf, E. V., \& Petre, R. 1999, in Proc. 26th Int. Cosmic Ray Conf., Salt Lake City, ed. D. Kieda, M. Salamon, \& B. Dingus, 3, 480

Bell, A. R., \& Lucek, S. G. 2001, MNRAS, 321, 433

Blandford, R. D., \& Eichler, D. 1987, Phys. Rep., 154, 1

Casse, F., \& Marcowith, A. 2003, A\&A, 404, 405

Drury, L. O. C. 1983, Rep. Prog. Phys., 46, 973

Drury, L. O. C., Markiewicz, W. J., \& Völk, H. J. 1989, A\&A, 225, 179

Duin, R. M., \& Strom, R. G. 1975, A\&A, 39, 33

Gardiner, C. W. 1983, Handbook of Stochastic Methods (Berlin: Springer Verslag), Chap. 4

Gotthelf, E. V., Koralesky, B., Rudnick, L., et al. 2001, ApJ, 552, L39

Hwang, U., Decourchelle, A., Holt, S. S., \& Petre, R. 2002, ApJ, 581, 1101

Jones, F. C. 1990, ApJ, 361, 162

Jones, F. C., \& Ellison, D. C. 1991, Space Sci. Rev., 58, 259

Kang, H., \& Jones, T. W. 1991, MNRAS, 249, 439

Kirk, J. G. 1994, in Plasma Astrophysics, Saas-Fee Advanced Course 24, ed. A. O. Benz, \& T. J.-L. Couvoisier, Springer Verlag, Berlin, 262

Koyama, K., Petre, R., Gotthelf, E. V., et al. 1995, Nature, 378, 255

Koyama, K., Kinugasa, K., Matsuzaki, K., et al. 1997, PASJ, 49, L7

Krülls, W. M., \& Achterberg, A. 1994, A\&A, 286, 314

LeVeque, R. J. 1998, in Computational Methods for Astrophysical Fluid Flow, Saas Fee Adv. Course 27, ed. R. J. LeVeque, D. Mihalas, E. A. Dorfi, \& E. Müller (Heidelberg: Springer Verlag), 1 Lucek, S. G., \& Bell, A. R. 2000, MNRAS, 314, 65

MacKinnon, A. L., \& Craig, I. J. D. 1991, A\&A, 251, 693

Marcowith, A., \& Kirk, J. G. 1999, A\&A, 347, 391

Mastichiadis, A., \& de Jager, O. C. 1996, A\&A, 311, L5

McKee, C. F., \& Truelove, J. K. 1995, Phys. Rep., 256, 157

Reynolds, S. P. 1998, ApJ, 493, 375

Reynolds, S. P., \& Chevalier, R. A. 1981, ApJ, 245, 912

Rybicki, G. B., \& Lightman, A. P. 1979, Radiative Processes in Astrophysics (New York: John Wiley \& Sons), Chap. 6

Saslaw, W. C. 1985, Gravitational physics of stellar and galactic systems (Cambridge Univ. Press), 18

Skilling, J. 1975, MNRAS, 172, 557

Slane, P., Gaensler, B. M., Dame, T. M., et al. 1999 ApJ, 525, 357

Slane, P., Hughes, J. P., Edgar, R. J., et al. 2001, ApJ, 548, 81

Tóth, G. 1996, Astrophys. Lett. \& Comm., 34, 245

Tóth, G., \& Odstrčil 1996, J. Comp. Phys., 128, 82

Vink, J., \& Laming, J. M. 2003, ApJ, 584, 758

Woltjer, L. 1972, ARA\&A, 10, 129

Zeldovich, Ya. B., Ruzmaikin, A. A., \& Sokoloff, D. D. 1983, Magnetic Fields in Astrophysics (Gordon and Breach Science Publishers), 75 